\author{
ARTIFICIAL SATELLITES, Vol. 43, No. 3 - 2008 \\ DOI: 10.2478/v10018-009-0011-x
}

\title{
THE EFFECT OF POLAR GAPS ON THE SOLUTIONS OF GRADIOMETRIC BOUNDARY VALUE PROBLEMS
}

\author{
M. Eshagh \\ Royal Institute of Technology, Division of Geodesy, Stockholm, Sweden \\ Fax:+46 87907343 ; Tel: +46 87907369 \\ Email:eshagh@kth se
}

\begin{abstract}
The lack of satellite gravity gradiometric data, due to inclined orbit, in the Polar Regions influences the geopotential coefficients obtained from the solutions of gradiometric boundary value problems. This paper investigates the polar gaps effect on these solutions and it presents that the near zero-, first- and second-order geopotential coefficients are weakly determined by the vertical-vertical, vertical-horizontal and horizontal solutions, respectively. Also it shows that the vertical-horizontal solution is more sensitive to the lack of data than the other solutions.
\end{abstract}

Keywords: satellite gravity gradiometry, associated Legendre function, loss of gravitational signal, tensor spherical harmonics

\section{INTRODUCTION}

The satellite orbits used in practice for the gravity field recovery missions are inclined causing the polar gaps in the data coverage. If the inclination of low orbiters is not equal to $90^{\circ}$, then the polar areas are not covered with satellite observations. The polar gaps problem was described by Rummel et al. (1993) and Koop (1993) with concentration on determining the spherical harmonic coefficients using the least-squares method. Sneeuw and van Gelderen (1997) also studied this problem and explained why the near-zonal harmonics are weakly determined in geopotential modeling. They have considered both space-wise and time-wise approaches in their discussions. Tscherning et al. (2000) investigated the polar gaps problem using least-squares collocation. They concluded that the inclusion of gravity data in the gaps will improve the estimates of the geopotential coefficients. Tscherning (2001) mentioned that the near-zonal harmonics were typically two times larger than the other harmonics if the poles were not covered. He found that if ground gravity data is used to fill-in the gaps, the data should have a resolution twice the second-order radial derivative $T_{z z}$. He concluded that the available airborne and sub-marine gravimetry data would not improve the gravity field and steady-state ocean circulation explorer (GOCE) (see e.g. Balmino et al. 1998 and 2001, ESA 1999, Albertella et al. 2002) solution if data from CHAMP (Challenging Minisatellite Payload) (Reigber et al. 1999 and 2004), and GRACE (Gravity Recovery and Climate Experiments) (Tapley et al. 2005) are available. Pail et al. (2001) used an orthonormalization scheme to orthonomalize the base functions with reference to Hwang (1991) and (1993). They recovered the geopotential coefficients without filling-in the gaps. Rudolph et al. (2002) considered the polar gaps filling-in problem from the satellite gradiometric data, they 
conclude that $1^{\circ} \times 1^{\circ}$ gravity anomaly blocks can be recovered. They also mentioned that the error of the recovered gravity anomalies reduced from 3 to $10-12 \mathrm{mGal}$ from the rim to the centre of gap. They found also the cross-track direction contain the most information. Metzler and Pail (2005) presented a spherical cap regularization method considering an analytical function, which is defined in the polar region using a geopotential model. Simons and Dahlen (2006) used the spherical Slepian function for the polar gaps, and they proposed a new method that expands the source field in terms of a truncated basis set of spherical Slepian function and compared it a with least-squares method. Siemes et al. (2007) incorporated three strategies: augmenting data in the gaps, introducing a priori information for regularization and the Slepian approach again.

In this paper, it is investigated which parts of the gravitational signal are not well estimated in the spectral solutions of gradiometric boundary value problems (GBVPs). Similar investigation was done by Sneeuw and Gelderen (1997) only for the vertical-vertical solution, which is simplest one among the other solutions of GBVPs. The other solutions have not been treated so far. Here, we want to present the polar gaps effects on these solutions.

\section{GRAVITATIONAL SIGNAL AND POLAR GAPS}

In the integral solutions of the GBVPs, combinations of the gravitational gradients are considered. The polar gaps destroy the unbiasedness of the solution, and some parts of the gravitational signal are weakly determined. The loss the gravitational signal due to polar gaps was investigated by Sneeuw and Gelderen (1997) by a simple integral approach on the vertical-vertical gradient $\left(T_{z z}\right)$. They found that the zonal and the near zonal geopotential coefficients are weakly estimated because of the polar gaps. Here we try to generalize this idea to the other solutions of GBVPs. Let us start with the spherical harmonic expansion of the disturbing potential

$$
T(P)=\frac{G M}{R} \sum_{n=2}^{\infty}\left(\frac{R}{r}\right)^{n+1} \sum_{m=-n}^{n} t_{n m} Y_{n m}(P),
$$

where $R$ is the semi-major axis of the reference ellipsoid, $r$ is the geocentric distance of the space-borne gradiometer (at satellite level). GM is the geocentric gravitational constant time of the Earth's mass, $P$ is the computation point with the co-latitude $\theta^{\prime}$ and the longitude $\lambda^{\prime}$, $t_{n m}$ are the spherical harmonic coefficient of $T$ with degree $n$ and order $m$ and $Y_{n m}(P)$ is the surface spherical harmonics. The solution of the harmonic coefficients according to orthogonality of the spherical harmonics

$$
\iint_{\sigma} Y_{n m}(Q) Y_{n^{\prime} m^{\prime}}(Q) d \sigma=4 \pi \delta_{n n^{\prime}} \delta_{m m^{\prime}}
$$

is:

$$
t_{n m}=\frac{1}{4 \pi} \iint_{\sigma} T(Q) Y_{n m}(Q) d \sigma
$$

where $Q$ is the integration point with the co-latitude $\theta$ and the longitude $\lambda, \delta$ stands for Kronecker's delta with following definition: 


$$
\delta_{i j}=\left\{\begin{array}{lll}
1 & \text { if } \quad i=j \\
0 & \text { if } & i \neq j
\end{array},\right.
$$

and $d \sigma$ is the horizontal integration element. Mathematically, the loss of gravitational signal can be expressed by Eq. (1c), which is a simple global spherical harmonic analysis of disturbing potential $(T)$ on the sphere $\sigma$. If a quadrature integral formula is used to compute the geopotential coefficients, a global coverage of satellite gradiometric data is required. However, the solution of the geopotential coefficients is biased due to the polar gaps. The following quadrature estimator of the geopotential coefficients considers the polar gaps in the global harmonic analysis:

$$
\hat{t}_{n m}=\frac{1}{4 \pi} \iint_{\sigma \backslash \sigma_{0}} T(Q) Y_{n m}(Q) d \sigma
$$

where $\hat{t}_{n m}$ is the biased estimate of the spherical harmonic coefficient of the disturbing potential with degree $n$ and order $m$ and $\sigma_{0}$ is the joint support of two polar gaps. In the case of satellite gradiometry, the observations are the gravitational gradients. Here we use the gradients expressed in the local north-oriented frame. The $z$-axis of the frame is pointing upwards in the geocentric radial direction, the $x$-axis towards the north and the $y$-axis is directed to the west to complete a right handed frame. Similar integral formulas to Eq. (2) can be constructed for the gradiometric observables. These formulas are the spectral solutions of the GBVPs and can be written as (see also Martinec 2003, Eshagh 2009):

$$
\begin{aligned}
& \hat{t}_{n m}=\frac{R^{3}}{4 \pi G M(n+1)(n+2)}\left(\frac{r}{R}\right)^{n+3} \iint_{\sigma \mid \sigma_{0}} T_{z z}(Q) Y_{n m}(Q) d \sigma \\
& \hat{t}_{n m}=\frac{R^{3}}{4 \pi G M(n+1)(n+2)}\left(\frac{r}{R}\right)^{n+3} \iint_{\sigma \mid \sigma_{0}}\left[T_{x z}(Q) E_{n m}(Q)+T_{y z}(Q) F_{n m}(Q)\right] d \sigma,
\end{aligned}
$$

and

$$
\begin{aligned}
\hat{t}_{n m} & =\frac{R^{3}}{4 \pi G M(n-1) n(n+1)(n+2)}\left(\frac{r}{R}\right)^{n+3} \iint_{\sigma \mid \sigma_{0}}\left[\left(T_{x x}(Q)-T_{y y}(Q)\right) G_{n m}(Q)+\right. \\
& \left.+2 T_{x y}(Q) H_{n m}(Q)\right] d \sigma,
\end{aligned}
$$

where $T_{x x}(Q), T_{y y}(Q), T_{z z}(Q), T_{x y}(Q), T_{x z}(Q)$ and $T_{y z}(Q)$ are the $2^{\text {nd }}$ derivatives of the disturbing potential $(T)$ at the integration point in the local frame. To begin with, let us define:

$$
Q_{m}(\lambda)=\left\{\begin{array}{ll}
\cos m \lambda & m \leq 0 \\
\sin m \lambda & m>0
\end{array},\right.
$$

which is involved with definition of the functions $Y_{n m}(Q), E_{n m}(Q), F_{n m}(Q), G_{n m}(Q)$ and $H_{n m}(Q)$ (Eshagh 2009) as:

$$
Y_{n m}(Q)=Q_{m}(\lambda) \bar{P}_{n m}^{\prime}(\theta)
$$




$$
\begin{aligned}
& E_{n m}(Q)=Q_{m}(\lambda) \bar{E}_{n m}^{\prime}(\theta), \\
& F_{n m}(Q)=Q_{-m}(\lambda) \bar{F}_{n m}^{\prime}(\theta), \\
& G_{n m}(Q)=Q_{m}(\lambda) \bar{G}_{n m}^{\prime}(\theta), \\
& H_{n m}(Q)=Q_{-m}(\lambda) \bar{H}_{n m}^{\prime}(\theta),
\end{aligned}
$$

where

$$
\begin{aligned}
\bar{E}_{n m}^{\prime}(\theta) & =\frac{\partial \bar{P}_{n|m|}(\theta)}{\partial \theta}=a_{n m}^{1} \bar{P}_{n,|m|-1}+a_{n m}^{2} \bar{P}_{n,|m|+1}, \\
\bar{F}_{n m}^{\prime}(\theta) & =m \frac{\bar{P}_{n|m|}}{\sin \theta}= \begin{cases}c_{n m}^{1} \bar{P}_{n-1,|m|-1}+c_{n m}^{2} \bar{P}_{n-1,|m|+1} & \text { (backward mode) } \\
c_{n m}^{\prime 1} \bar{P}_{n+1,|m|-1}+c_{n m}^{\prime 2} \bar{P}_{n+1,|m|+1} & \text { (forward mode) },\end{cases} \\
\bar{G}_{n m}^{\prime}(\theta) & =\left(\frac{\partial^{2} \bar{P}_{n|m|}}{\partial \theta^{2}}-\cot \theta \frac{\partial \bar{P}_{n|m|}}{\partial \theta}+m^{2} \frac{\bar{P}_{n|m|}}{\sin ^{2} \theta}\right)= \\
& =p_{n m}^{1} \bar{P}_{n,|m|-2}+p_{n m}^{2} \bar{P}_{n|m|}+p_{n m}^{3} \bar{P}_{n,|m|+2}, \\
\bar{H}_{n m}^{\prime}(\theta) & =2 m \frac{\partial}{\partial \theta}\left(\frac{\bar{P}_{n|m|}}{\sin \theta}\right)=\left\{\begin{array}{ll}
q_{n m}^{1} \bar{P}_{n-1,|m|-1}+q_{n m}^{2} \bar{P}_{n-1,|m|+1} & \text { (backward mode) } \\
q_{n m}^{\prime 1} \bar{P}_{n+1,|m|-1}+q_{n m}^{\prime 2} \bar{P}_{n+1,|m|+1} & \text { (forward mode) }
\end{array} .\right.
\end{aligned}
$$

The constant coefficients $a_{n m}^{1}, a_{n m}^{2}, c_{n m}^{1}, c_{n m}^{2}, c_{n m}^{\prime 1}, c_{n m}^{\prime 2}, p_{n m}^{1}, p_{n m}^{2}, p_{n m}^{3}, q_{n m}^{1}, q_{n m}^{2}, q_{n m}^{3}$, $q_{n m}^{\prime 1}, q_{n m}^{\prime 2}$ and $q_{n m}^{\prime 3}$ are given in the appendix. Equations (4f)-(4i) shows the relation between the functions $\bar{E}_{n m}^{\prime}(\theta), \bar{F}_{n m}^{\prime}(\theta), \bar{G}_{n m}^{\prime}(\theta)$ and $\bar{H}_{n m}^{\prime}(\theta)$ and the associated Legendre function (ALF) $\bar{P}_{n|m|}(\theta)$. The last part of these equations were simplified by Eshagh (2009, Chapter 2) which are beneficial for numerical computations as they do not involve the first- and/or second-order derivative of the ALFs and they are non-singular at the poles. The last part of Eqs. (4g) and (4i) are two alternative equations for expression of $F_{n m}^{\prime}(\theta)$ and $H_{n m}^{\prime}(\theta)$, which means that either the forwarded mode with respect to $n$ i.e. $n+1$ or backwarded mode $n-1$ can be used.

Equations (3a), (3b) and (3c) were named by Martinec (2003) vertical-vertical, verticalhorizontal and horizontal-horizontal solutions of the GBVP. Theoretically these equations yield the same result, but because of discretization error of integrals and the noise of the gradiometric data, they do not in practice. All these equations can be used for gravity field recovery but the quality of the geopotential coefficients will not be the same. For more details about this matter the reader is referred to Eshagh (2009, Chapter 2). Also an optimal way of combination for these solutions are found in Eshagh (2009, Chapter 6).

\section{ALTERNATIVE EXPRESSIONS FOR $\boldsymbol{E}_{n m}^{\prime}(\theta), \boldsymbol{F}_{n m}^{\prime}(\theta), \boldsymbol{G}_{n m}^{\prime}(\theta)$ AND $\boldsymbol{H}_{n m}^{\prime}(\theta)$}

The functions $\bar{P}_{n m}^{\prime}(\theta), \bar{E}_{n m}^{\prime}(\theta), \bar{F}_{n m}^{\prime}(\theta), \bar{G}_{n m}^{\prime}(\theta)$ and $\bar{H}_{n m}^{\prime}(\theta)$ presented in Eqs. (4f)-(4i) are fully-normalized. Here we discuss on the unnormalized ones, i.e. $P_{n m}^{\prime}(\theta), E_{n m}^{\prime}(\theta)$, $F_{n m}^{\prime}(\theta), G_{n m}^{\prime}(\theta)$ and $H_{n m}^{\prime}(\theta)$ because the consequence of the study will be the same as the fully-normalized ones, as the normalization just reduces the magnitude of a function and the 
other properities of the function are preserved. The relation between the fully-normalized and unnormalized ALFs are given in e.g. Heiskanen and Moritz (1967, Eq. 1-73, p. 31). Now let the following formula which expresses the relation between the ALFs and the Legendre polynomials (Heiskanen and Moritz 1967, p.23):

$$
P_{n m}(\cos \theta)=\sin ^{m} \theta \frac{d^{m} P_{n}(\cos \theta)}{d(\cos \theta)^{m}} \text {. }
$$

Equation (4f) shows that $E_{n m}^{\prime}(\theta)$ is the first-order derivative of the ALFs with respect to $\theta$. Then differentiating Eq. (5) with respect to this parameter is:

$$
E_{n m}^{\prime}(\theta)=m \cos \theta \sin ^{m-1} \theta \frac{d^{m} P_{n}(\cos \theta)}{d(\cos \theta)^{m}}-\sin ^{m+1} \theta \frac{d^{m+1} P_{n}(\cos \theta)}{d(\cos \theta)^{m+1}} .
$$

According to Eqs. (4g) and (5) we obtain:

$$
F_{n m}^{\prime}(\theta)=m \sin ^{m-1} \theta \frac{d^{m} P_{n}(\cos \theta)}{d(\cos \theta)^{m}}
$$

The situation for $G_{n m}^{\prime}(\theta)$ is not as simple as that for $E_{n m}^{\prime}(\theta)$ and $F_{n m}^{\prime}(\theta)$, because $G_{n m}^{\prime}(\theta)$ consists of three terms. First we simplify the first-term of Eq. (4h) which is the second-order derivative of the ALFs with respect to $\theta$. Differentiation of Eq. (6a) with respect to $\theta$ yields:

$$
\begin{aligned}
\frac{d^{2} P_{n m}(\cos \theta)}{d \theta^{2}} & =\left[-m \sin ^{m} \theta+m(m-1) \cos ^{2} \theta \sin ^{m-2} \theta\right] \frac{d^{m} P_{n m}(\cos \theta)}{d(\cos \theta)^{m}}- \\
& -(2 m+1) \cos \theta \sin ^{m} \theta \frac{d^{m+1} P_{n}(\cos \theta)}{d(\cos \theta)^{m+1}}+\sin ^{m+2} \theta \frac{d^{m+2} P_{n}(\cos \theta)}{d(\cos \theta)^{m+2}}
\end{aligned}
$$

The second-term in the parenthesis in Eq. (4h) is the multiplication of $\cot \theta$ and the firstorder derivative of ALFs. It can be simplified to:

$$
\cot \theta \frac{d P_{n m}(\cos \theta)}{d \theta}=m \cos ^{2} \theta \sin ^{m-2} \theta \frac{d^{m} P_{n}(\cos \theta)}{d(\cos \theta)^{m}}-\cos \theta \sin ^{m} \theta \frac{d^{m+1} P_{n}(\cos \theta)}{d(\cos \theta)^{m+1}},
$$

and the last terms will be constructed by multiplication of $m / \sin \theta$ to Eq. (6b). Summation of Eqs. (6c) and (6d) and $m / \sin \theta$ time of Eq. (6b) yields:

$$
\begin{aligned}
G_{n m}^{\prime}(\theta) & =m\left(\left[(m-2) \cos ^{2} \theta+m\right] \sin ^{m-2} \theta-\sin ^{m} \theta\right) \frac{d^{m} P_{n}(\cos \theta)}{d(\cos \theta)^{m}}- \\
& -2 m \cos \theta \sin ^{m} \theta \frac{d^{m+1} P_{n}(\cos \theta)}{d(\cos \theta)^{m+1}}+\sin ^{m+2} \theta \frac{d^{m+2} P_{n}(\cos \theta)}{d(\cos \theta)^{m+2}}
\end{aligned}
$$


According to Eqs. (4i) and (5) it is easy to show that

$$
H_{n m}^{\prime}(\theta)=2 m\left[(m-1) \cos \theta \sin ^{m-2} \theta \frac{d^{m} P_{n}(\cos \theta)}{d(\cos \theta)^{m}}-\sin ^{m} \theta \frac{d^{m+1} P_{n}(\cos \theta)}{d(\cos \theta)^{m+1}}\right]
$$

The question is, what the advantages of these expressions are. These expressions are not practically useful but they help us to interpret the solutions of GBVPs. We start the discussion with Eq. (5) which is the definition of the ALFs. The Legendre polynomial $P_{n}(\cos \theta)$ in the right hand side of this equation has roots between -1 and 1 and the derivative, $d^{m} P_{n}(\cos \theta) / d(\cos \theta)^{m}$ is a polynomial of degree $n-m$ with $n-m$ roots. The factor $\sin ^{m} \theta$ will give more weights to the area around the equator by increasing $m$ (Sneeuw and Gelderen 1997). Reversely the weights decrease by reducing the order $m$ and when $m=0$, it is minimum. It implies that the weights on the poles are maxima for the zonal terms. The mathematical derivations for $E_{n m}^{\prime}(\theta)$ and $F_{n m}^{\prime}(\theta)$ show that the weighting factor for these two functions is $\sin ^{m-1} \theta$ and when $m=1$ the factor gives the lowest weights for the equatorial areas, and the most weights for the polar region. Since Eq. (3b), which is the solution of the vertical-horizontal GBVP, involved with these functions, it yields weak solution for the harmonics $\hat{t}_{n 1}$, or the first-order harmonics. In a very similar way, we can see that $G_{n m}^{\prime}(\theta)$ and $H_{n m}^{\prime}(\theta)$ involve the factor $\sin ^{m-2} \theta$ and Eq. (3c), which is the solution of the horizontal-horizontal GBVP, delivers weak second-order harmonic coefficients $\left(\hat{t}_{n_{2}}\right)$.

One may think that it is not enough to consider the factors $\sin ^{m} \theta, \sin ^{m-1} \theta$ and $\sin ^{m-2} \theta$ for interpretation of the results, because some of the expressions contain other factors which are function of the order $m, m+1$ and/or $m-1$ as well. Here, we numerically investigate this matter to see if the numerical results support the above explanations. The following section investigates the functions $E_{n m}^{\prime}(\theta), F_{n m}^{\prime}(\theta), G_{n m}^{\prime}(\theta)$ and $H_{n m}^{\prime}(\theta)$ at different co-latitudes, degrees and orders.

\section{NUMERICAL STUDIES}

In order to perform a numerical study on the functions $P_{n m}^{\prime}(\theta), E_{n m}^{\prime}(\theta), F_{n m}^{\prime}(\theta), G_{n m}^{\prime}(\theta)$ and $H_{n m}^{\prime}(\theta)$, we consider the constant degree $n=10$ and plot the functions for the orders $m=$ $0,1,2,3,4$ and 5 , respectively in co-latitudes from $0^{\circ}$ to $180^{\circ}$. In Fig. 1 the solid-tick line shows the order in which the value of the function is maximal at the poles. The figure confirms the explanation made in the previous section. As we see $P_{n m}^{\prime}(\theta)$ selects its maxima at the poles when $m=0, E_{n m}^{\prime}(\theta)$ and $F_{n m}^{\prime}(\theta)$ when $m=1$ and $G_{n m}^{\prime}(\theta)$ and $H_{n m}^{\prime}(\theta)$ when $m$ $=2$. In order to see how these functions behave in different degrees, the constant order $m=10$ is considered and the functions are plotted in different co-latitudes and the degrees $n=10,20$, 30, 40 and 50. The results of the computations are plotted in Fig. 2. As the figure shows when the degree $n$ increases the functions go further to the poles. 

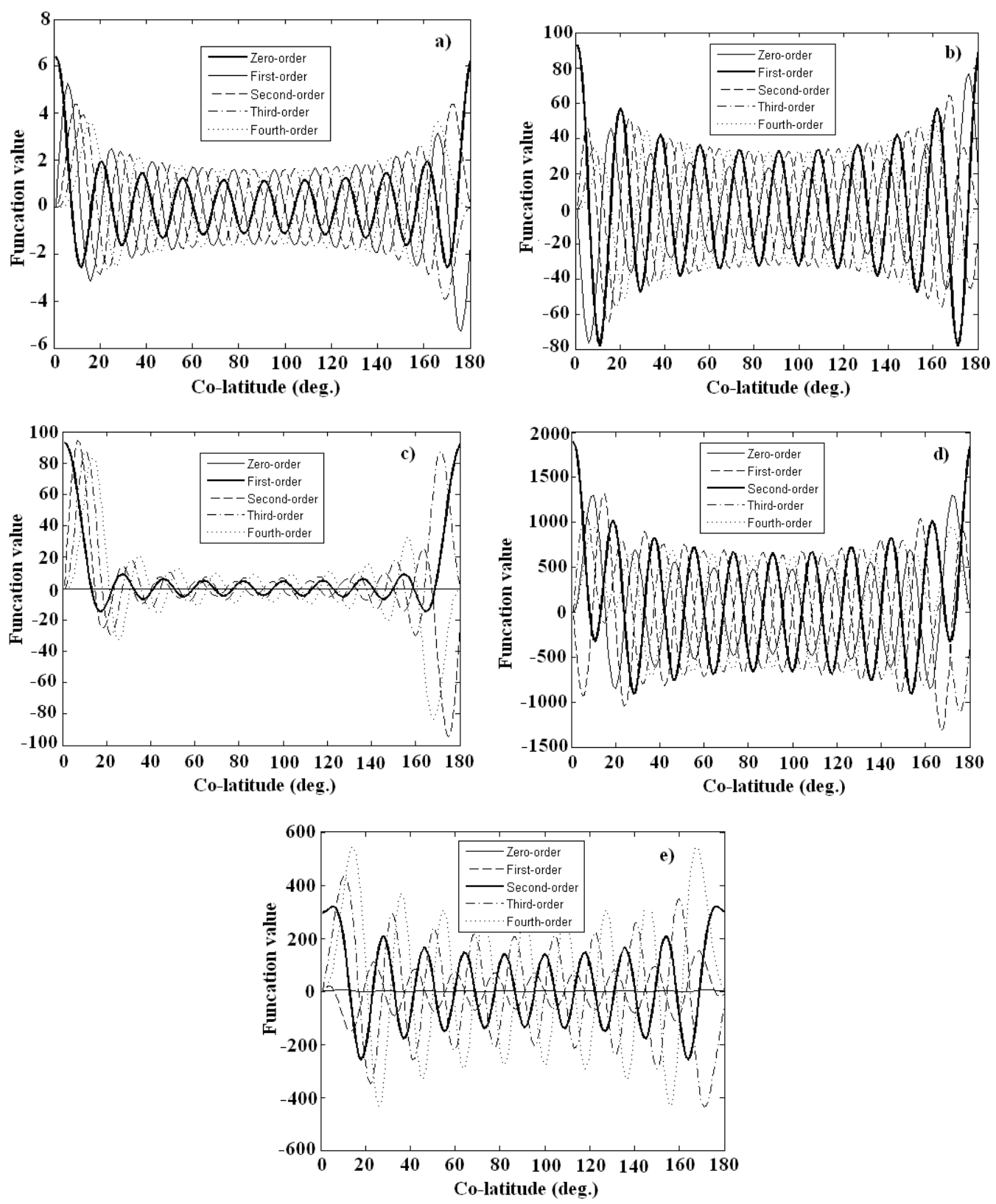

Fig. 1. Values of a) $P_{n m}^{\prime}(\theta)$, b) $E_{n m}^{\prime}(\theta)$, c) $F_{n m}^{\prime}(\theta)$, d) $G_{n m}^{\prime}(\theta)$ and e) $H_{n m}^{\prime}(\theta)$ at different co-latitudes with $n=10$ and $m=0,1,2,3,4$ and 5

What we learn from Figs. (2) and (3) is that the high degrees are more sensitive to the polar gaps in the solutions of GBVPs. In the vertical-vertical, vertical-horizontal and horizontalhorizontal solutions, the near order 0,1 and 2 geopotential coefficients are weakly determined, respectively. 

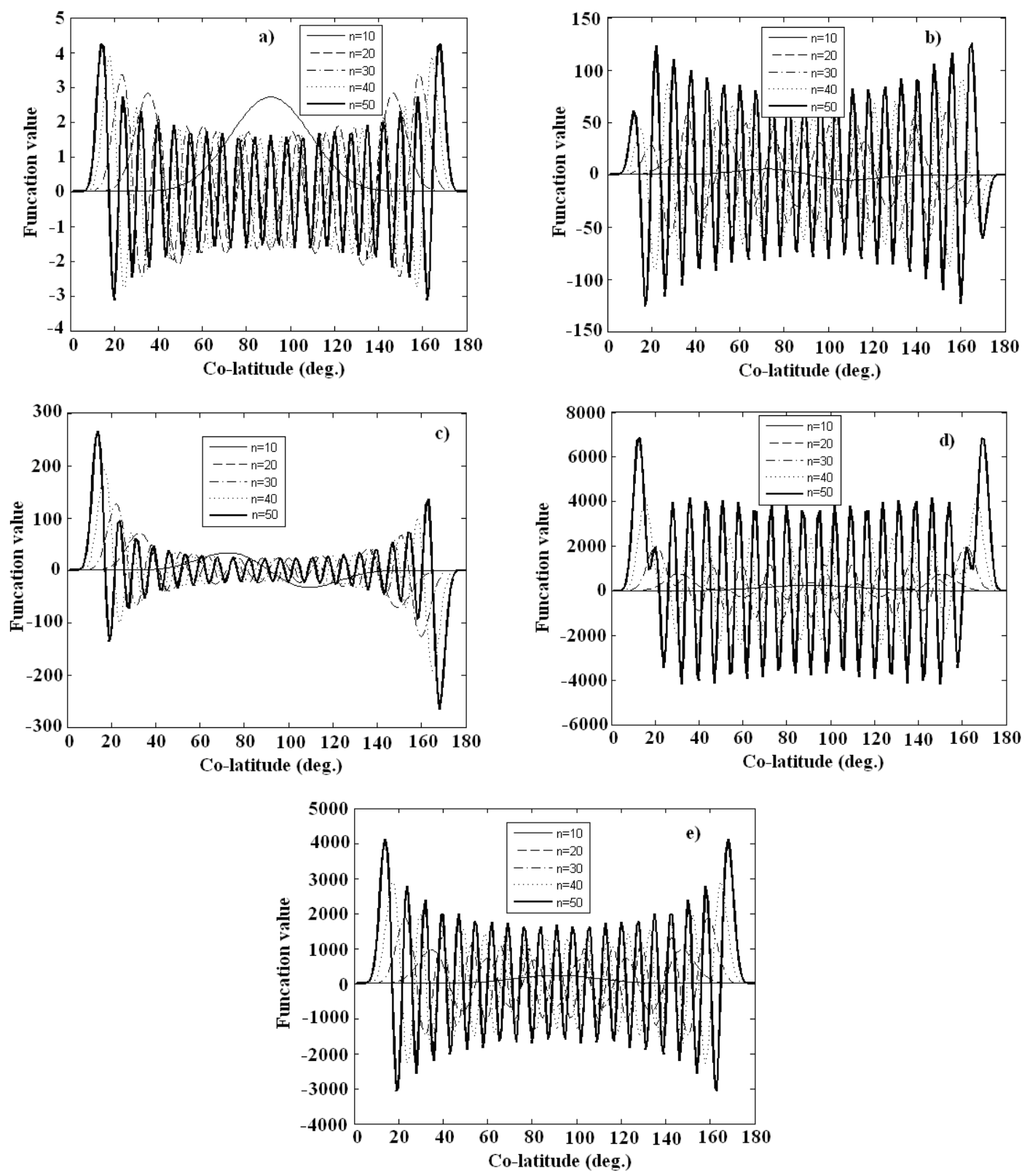

Fig. 2. Values of a) $P_{n m}^{\prime}(\theta)$, b) $E_{n m}^{\prime}(\theta)$, c) $F_{n m}^{\prime}(\theta)$, d) $G_{n m}^{\prime}(\theta)$ and e) $H_{n m}^{\prime}(\theta)$ at different co-latitudes with $m=10$ and $n=10,20,30,40$ and 50

In order to study the loss of the gravitational signal numerically, the EGM96 (Lemoine et al. 1998) is used to generate the gravitational gradients at satellite level $(250 \mathrm{~km})$ with $0.5^{\circ} \times 0.5^{\circ}$ resolution using the Eshagh (2009) expressions for the gradients. The relative error of the harmonics is $\varepsilon_{n m}=\left(\hat{t}_{n m}-t_{n m}\right) / t_{n m}$, which could be a criterion to compare the solutions of GBVPs. $\hat{t}_{n m}$ is estimated from Eqs. (3a)-(3c) and $t_{n m}$ is the true values of harmonic coefficients. We assume the harmonics of EGM96 as the true values in this study. The $7^{\circ}$ polar gaps is considered in the coverage of satellite gradiometric data. The north and south polar gaps are the regions at latitudes above $83^{\circ}$ and below $-83^{\circ}$, respectively. In this case a 
matrix with the same size of the gradients' grid is generated in which the elements placed in the polar gaps are zero and the others are 1. This matrix is multiplied to the matrix of the gradients element-wisely. The result will be the matrices of gradients with zero upper and lower cells. In other words, we use the following matrix of the gradiometric data.

$$
\left[\begin{array}{c}
\mathbf{0} \\
\mathbf{T}_{i j}^{2} \\
\mathbf{0}
\end{array}\right]=\left[\begin{array}{c}
\mathbf{T}_{i j}^{1} \\
\mathbf{T}_{i j}^{2} \\
\mathbf{T}_{i j}^{3}
\end{array}\right] \#\left[\begin{array}{c}
\mathbf{0} \\
\mathbf{J} \\
\mathbf{0}
\end{array}\right],
$$

where 0 is a 14 by 720 null matrix which is equivalent to size of the polar gaps according to $0.5^{\circ} \times 0.5^{\circ}$ resolution, $\mathbf{J}$ is a 332 by 720 matrix and all its elements are 1 . \# stands for elementwise product which means that each element of a matrix is multiplied to its corresponding element in another matrix (with the same dimensions). $\mathbf{T}_{i j}(i, j=x, y$ and $z$ ) stands for the representer matrix of the gradients which is separated into three parts. Two parts of this matrix are placed on the polar gaps, i.e. $\mathbf{T}_{i j}^{1}$ and $\mathbf{T}_{i j}^{3}$ which are element-wisely multiplied to $\mathbf{0}$. According to Eq. (7) the matrices of the gradients are inserted into Eqs. (3a)-(3c) to estimate the geopotential coefficients.
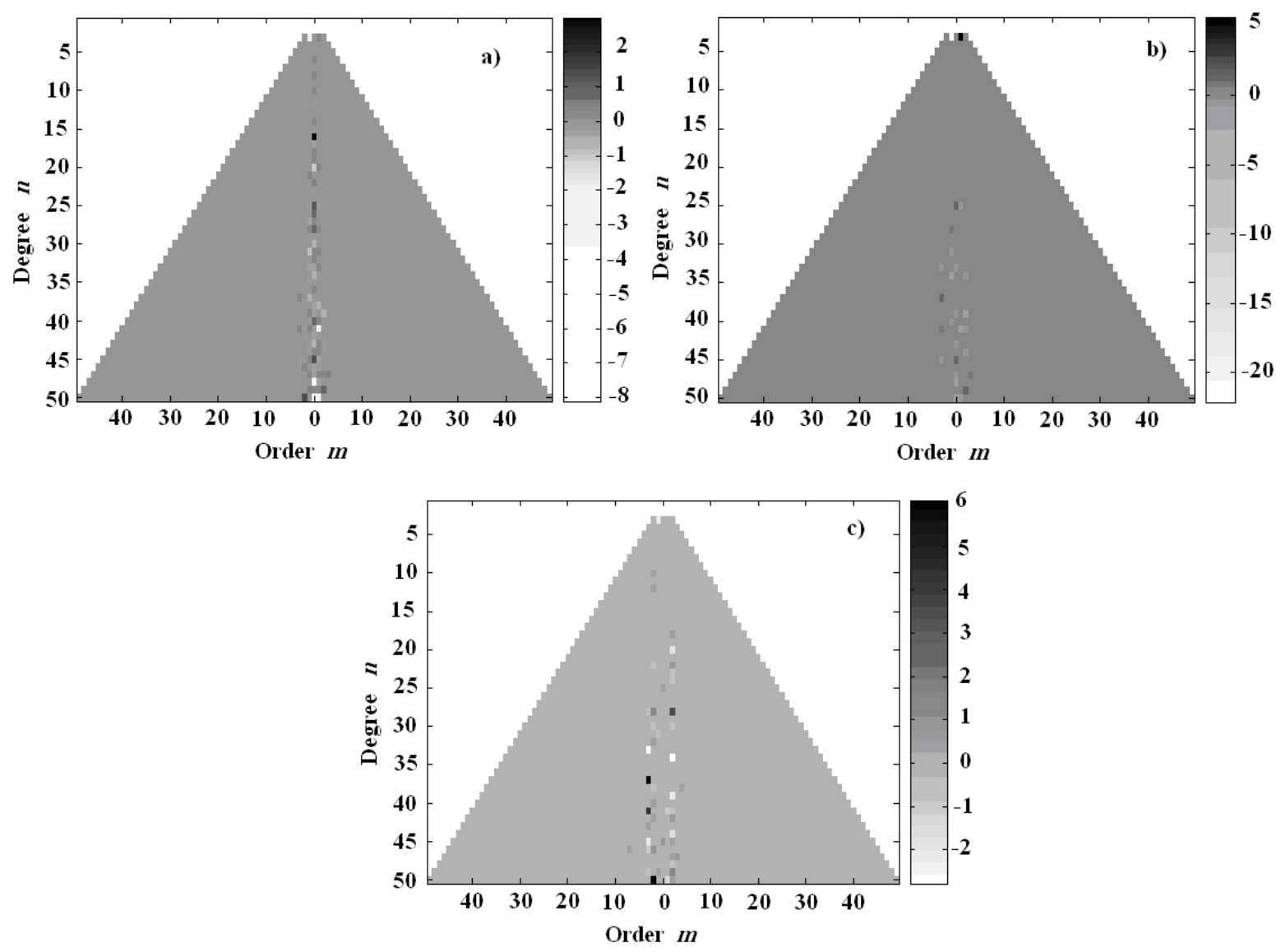

Fig. 3. Relative error of geopotential coefficients obtained from a) vertical-vertical, b) vertical-horizontal and c) horizontal-horizontal GBVP 
Figure 3 shows the triangle spectra of the sine and cosine coefficients below degree and order 50. The left part of each triangle shows the cosine and the right one the sine geopotential coefficients. The figure shows that the near zonal, first- and second-order coefficients are influenced by the polar gaps. It can be seen that the vertical-horizontal gradients are the most influenced gradients. Figure 3 confirms the explanation made in Section 3 as well.

\section{CONCLUSIONS}

We theoretically showed that the geopotential coefficients around the orders 0,1 and 2 derived from the vertical-vertical, vertical-horizontal and horizontal-horizontal solutions, respectively are biased. The polar gaps influence high degrees in all the solutions and the loss of the gravitational signal is higher for the vertical-horizontal solution than the other solutions.

Acknowledgments. The author is thankful to Professor Lars E. Sjöberg for scientific discussion about the polar gaps. The Swedish National Space Board is appreciated for the financial support project No. 63/07:1. Dr. G. Strykowski is cordially appreciated for the constructive comments and the quick review. Mr. M. Gadomski is also appreciated for his helps.

\section{APPENDIX A}

The constant coefficients of Eqs. (4a)-(4f) (Eshagh 2009):

$$
\begin{aligned}
& a_{n m}^{1}=\frac{1}{2} \sqrt{n+|m|} \sqrt{n-|m|+1} \sqrt{\left(2-\delta_{|m| 0}\right) /\left(2-\delta_{|m|-1,0}\right)}, \\
& a_{n m}^{2}=-\frac{1}{2} \sqrt{n-|m|} \sqrt{n+|m|+1} \sqrt{\left(2-\delta_{|m| 0}\right) /\left(2-\delta_{|m|+1,0}\right)} \text {, } \\
& c_{n m}^{1}=\frac{m}{2|m|} \sqrt{n+|m|} \sqrt{n+|m|-1} \sqrt{\left(2-\delta_{|m| 0}\right)(2 n+1) /\left(2-\delta_{|m|-1,0}\right) /(2 n-1)}, \\
& c_{n m}^{2}=\frac{m}{2|m|} \sqrt{n-|m|} \sqrt{n-|m|-1} \sqrt{\left(2-\delta_{|m| 0}\right)(2 n+1) /\left(2-\delta_{|m|+1,0}\right) /(2 n-1)}, \\
& c_{n m}^{\prime 1}=\frac{m}{2|m|} \sqrt{n-|m|+1} \sqrt{n-|m|+2} \sqrt{\left(2-\delta_{|m| 0}\right)(2 n+1) /\left(2-\delta_{|m|-1,0}\right) /(2 n+3)} \text {, } \\
& c_{n m}^{\prime 2}=\frac{m}{2|m|} \sqrt{n+|m|+1} \sqrt{n+|m|+2} \sqrt{\left(2-\delta_{|m| 0}\right)(2 n+1) /\left(2-\delta_{|m|+1,0}\right) /(2 n+3)} \\
& p_{n m}^{1}=\frac{1}{2} \sqrt{\frac{2-\delta_{|m| 0}}{2-\delta_{|m|-2,0}}} \sqrt{n+|m|} \sqrt{n+|m|-1} \sqrt{n-|m|+1} \sqrt{n-|m|+2}, \\
& p_{n m}^{2}=m^{2} \text {, } \\
& p_{n m}^{3}=\frac{1}{2} \sqrt{\frac{2-\delta_{|m| 0}}{2-\delta_{|m|+2,0}}} \sqrt{n-|m|} \sqrt{n-|m|-1} \sqrt{n+|m|+1} \sqrt{n+|m|+2}, \\
& q_{n m}^{1}=\frac{m}{2|m|} \sqrt{\frac{\left(2-\delta_{|m| 0}\right)(2 n+1)}{\left(2-\delta_{|m|-2,0}\right)(2 n+3)}} \sqrt{n+|m|} \sqrt{n-|m|+3} \sqrt{n-|m|+2} \sqrt{n-|m|+1},
\end{aligned}
$$




$$
\begin{aligned}
& q_{n m}^{2}=\frac{m}{2|m|} \sqrt{\frac{(2 n+1)(n+|m|+1)}{(2 n+3)(n-|m|+1)}}[(n+|m|+2)(n-|m|+1)-(n-|m|+2)(n-|m|+1)] \\
& q_{n m}^{3}=-\frac{m}{2|m|} \sqrt{\frac{\left(2-\delta_{|m| 0}\right)(2 n+1)}{\left(2-\delta_{|m|+2,0}\right)(2 n+3)}} \sqrt{n-|m|} \sqrt{n+|m|+3} \sqrt{n+|m|+2} \sqrt{n+|m|+1}, \\
& q_{n m}^{\prime \prime}=\frac{m}{2|m|} \sqrt{\frac{\left(2-\delta_{|m| 0}\right)(2 n+1)}{\left(2-\delta_{|m|-2,0}\right)(2 n-1)}} \sqrt{n+|m|} \sqrt{n+|m|-3} \sqrt{n+|m|-2} \sqrt{n-|m|+1}, \\
& q_{n m}^{\prime 2}=-m \sqrt{\frac{(2 n+1)}{(2 n-1)} \sqrt{n-|m|} \sqrt{n+|m|}} \\
& q_{n m}^{\prime 3}=-\frac{m}{2|m|} \sqrt{\frac{\left(2-\delta_{|m| 0}\right)(2 n+1)}{\left(2-\delta_{|m|+2,0}\right)(2 n-1)}} \sqrt{n-|m|} \sqrt{n-|m|-1} \sqrt{n-|m|-2} \sqrt{n+|m|+1} .
\end{aligned}
$$

\section{REFERENCES}

Albertella A., Migliaccio F., and Sansò F. (2002) GOCE: The Earth Field by Space Gradiometry. Celestial Mechanics and Dynamical Astronomy, Vol. 83, 1-15.

Balmino G., Perosanz F., Rummel R., Sneeuw N., Sünkel H. and Woodworth P., (1998) European Views on Dedicated Gravity Field Missions: GRACE and GOCE. An Earth Sciences Division Consultation Document, ESA, ESD-MAG-REP-CON-001.

Balmino G., Perosanz F., Rummel R., Sneeuw N. and Suenkel H. (2001) CHAMP, GRACE and GOCE: Mission Concepts and Simulations. Boll. Geof. Teor. Appl., Vol. 40, No. 3-4, 309-320.

ESA (1999) Gravity Field and Steady-State Ocean Circulation Mission, ESA SP-1233(1), Report for mission selection of the four candidate earth explorer missions. ESA Publications Division, pp. 217, July 1999.

Eshagh M. (2009) On satellite gravity gradiometry, Doctoral thesis in geodesy, Royal Institute of Technology (KTH), Stockholm, Sweden.

Heiskanen W. and Moritz H. (1967) Physical Geodesy. W.H Freeman and company, San Fransisco and London.

Hwang C. (1991) Orthogonal functions over the oceans and applications to the determination of orbit error, geoid and sea surface topography from satellite altimetry, $\mathrm{PhD}$ dissertation, $229 \mathrm{pp}$.

Hwang C. (1993) Fast algorithm for the formulation of normal equations in a least-squares spherical harmonic analysis by FFT, Manuscripta Geodaetica. 62:46-52.

Koop R. (1993) Global gravity field modeling using satellite gravity gradiometry. Publ Geodesy, New series, No. 38. Netherland Geodetic Commission, Delft.

Lemoine F.G., Kenyon S.C., Factor J.K., Trimmer R.G., Pavlis N.K., Chinn D.S., Cox C.M., Klosko S.M., Luthcke S.B., Torrence M.H., Wang Y.M., Williamson R.G., Pavlis E.C., Rapp R.H. and Olson T.R. (1998) The Development of the Joint NASA GSFC and NIMA Geopotential Model EGM96,_NASA/TP-1998-206861. Goddard Space Flight Center, Greenbelt

Martinec Z. (2003) Green's function solution to spherical gradiometric boundary-value problems, Journal of Geodesy, 77:41-49. 
Metzler B. and Pail R. (2005), GOCE data processing: the spherical cap regularization approach, Studia Geophysica et Geodaetica, 49: 441-462.

Pail R., Plank G. and Schuh W.D. (2001) Spatially restricted data distributions on the sphere: the method of orthonormalized functions and applications, Journal of Geodesy,. 75:44-56.

Reigber C., Schwintzer P. and Lühr H. (1999) The CHAMP geopotential mission, Boll. Geof. Teor. Appl. Vol. 40, 285-289.

Reigber Ch., Jochmann H., Wünsch J., Petrovic S., Schwintzer P., Barthelmes F., Neumayer K.-H., König R., Förste Ch., Balmino G., Biancale R., Lemoine J.-M., Loyer S. and Perosanz F. (2004) Earth Gravity Field and Seasonal Variability from CHAMP. In: Reigber, Ch., Lühr, H., Schwintzer, P., Wickert, J. eds. Earth Observation with CHAMP Results from Three Years in Orbit, Springer, Berlin, 25-30.

Rudolph S., Kusche J. and Ilk K. H. (2002) Investigations on the polar gap problem in ESA's gravity field and steady-state ocean circulation explorer mission (GOCE), Journal of Geodynamics, 33:65-74.

Rummel R., Sanso F., Gelderen M., Koop R., Schrama E., Brovelli M., Migiliaccio F., and Sacerdote F. (1993) Spherical harmonic analysis of satellite gradiometry. Publ Geodesy, New Series, No. 39 Netherlands Geodetic Commission, Delft.

Siemes C., Schuh W.D. Cai J., Sneeuw N. and Baur O. (2007) GOCE data processing: the numerical challenge of data gaps, Satus sem., observation of system Earth from space geotechnologien, science report 11, p.99-105, Munchen, Germany.

Simons F. J. and Dahlen F. A. (2006) Spherical Slepian functions and the polar gap in geodesy, Geophysical Journal International, 166:1039-1061.

Sneeuw N. and van Gelderen M. (1997) The polar gaps, In Lecture notes in Earth sciences, geodetic boundary value problems in view of the one centimeter geoid, Lecture notes in Earth Sciences eds. Sanso F and Rummel R, 2003, 559-568. Springer-Verlag

Tapley B., Ries J. Bettadpur S., Chambers D., Cheng M., Condi F., Gunter B., Kang Z., Nagel P., Pastor R., Pekker T., Poole S. and Wang F. (2005) GGM02-An improved Earth gravity field model from GRACE. Journal of Geodesy, Vol. 79, 467-478.

Tscherning C.C., Forsberg R., Albertella A., Migliaccio F. and Sanso F. (2000) The polar gap problem, Space-wise approaches to gravity field determination in Polar areas, Eötvös to mGal, Finsal report, 10 April 2000, Suenkel H. (Ed.), p. 331-336. Study team 2, UCPH, POLIMI, http://www.gfy.ku.dk/ cct/publ cct/cct1600.pdf.

Tscherning C. C. (2001) Covering the GOCE mission polar data gaps using gradients and ground gravity, In Proc. International GOCE user workshop, ESTEC, 23-24 April 2001, p. 105-110. ESA WPP-188.

Received: 2009-05-14,

Reviewed: 2009-06-03, by G. Strykowski,

Accepted: 2009-07-01. 